\title{
ANÁLISE DE CUSTOS DO TRATAMENTO DE LESÃO POR PRESSÃO EM PACIENTES INTERNADOS
}

\section{COST ANALYSIS OF THE TREATMENT OF PRESSURE INJURY IN HOSPITALIZED PATIENTS}

\section{ANÁLISIS DE COSTOS DEL TRATAMIENTO DE LESIÓN POR PRESIÓN EN PACIENTES INTERNADOS}

Miguir Terezinha Vieccelli Donoso ${ }^{1}$, Sara Almeida Souza Barbosa ${ }^{2}$, Giovana Paula Resende Simino ${ }^{3}$, Braulio Roberto Gonçalves Marinho Couto ${ }^{4}$, Flavia Falci Ercole ${ }^{5}$, Jaqueline Almeida Guimarães Barbosa ${ }^{6}$.

\section{RESUMO}

Objetivo: Avaliar o custo do tratamento de lesões por pressão em pacientes internados, relacionado a materiais de curativos e mão de obra do profissional de enfermagem. Método: estudo transversal, descritivo, com dados secundários de análise de custos diretos com materiais de curativos no tratamento de lesões por pressão, realizado em hospital de médio porte, de ensino, no estado de Minas Gerais. A amostra foi não probabilística, totalizando 60 participantes. Resultados: em relação aos custos com tratamento de lesão por pressão, observou-se média de custo semestral de $\mathrm{R} \$ 1.886,00$ por paciente e, custo total de R\$ 113.186,00. Quanto ao custo com profissionais da enfermagem, a maioria dos pacientes teve custo máximo de $\mathrm{R} \$ 1.000$. Conclusões: a avaliação da população e dos custos indicou que o tratamento de lesão por pressão acarreta custos elevados e onera as instituições de saúde. Medidas preventivas, quando possível, devem ser otimizadas, resultando em economia para a instituição. Descritores: Úlcera por Pressão; Economia da enfermagem; Enfermagem.

\section{ABSTRACT}

Objective: To evaluate the cost of treatment of pressure injuries in hospitalized patients, related to the dressing materials and nursing workforce. Method: cross-sectional, descriptive study with secondary data of direct cost analysis with dressing materials in the treatment of pressure lesions, conducted at a medium sized teaching hospital in the state of Minas Gerais. The sample was not probabilistic, totaling 60 participants. Results: in relation to costs with treatment of pressure injury, we observed a half-yearly cost average of $R \$ 1,886.00$ per patient, and a total cost of $R \$ 113,186.00$. As for the cost of nursing professionals, the majority of patients had a maximum cost of $R \$$ 1,000. Conclusions: the evaluation of population and costs indicated that the treatment of pressure injury entails high costs and burden health institutions. Preventive measures, when possible, should be optimized, resulting in savings for the institution.

Descriptors: Pressure Ulcer; Economics Nursing; Nursing.

\section{RESUMEN}

Objetivo: Evaluar el costo del tratamiento de lesiones por presión en pacientes hospitalizados, relacionado a materiales de curativos y mano de obra del profesional de enfermería. Método: Estudio transversal, descriptivo, con datos secundarios de análisis de costos directos con materiales de curativos en el tratamiento de lesiones por presión, realizado en un hospital docente de tamaño mediano en el estado de Minas Gerais. La muestra fue no probabilística, totalizando 60 participantes. Resultados: en relación a los costos con tratamiento de lesión por presión, se observó promedio de costo semestral de $R \$ 1.886,00$ por paciente $y$, costo total de $R$ \$ $113.186,00$. En cuanto al costo con profesionales de la enfermería, la mayoría de los pacientes tuvo un costo máximo de $R \$ 1.000$. Conclusiones: la evaluación de la población y de los costos indicó que el tratamiento de lesión por presión conlleva costos elevados y las instituciones de salud. Las medidas preventivas, cuando sea posible, deben ser optimizadas, resultando en economía para la institución.

Descriptores: Úlcera por Presión; Economía de la Enfermería; Enfermería.

\footnotetext{
${ }^{1}$ Doutora em Ciências da Saúde pela Faculdade de Medicina da UFMG. Docente do Departamento de Enfermagem Básica da Escola de Enfermagem da UFMG. ${ }^{2}$ Estomaterapeuta pela Escola de Enfermagem da UFMG. Enfermeira do Instituto de Previdência dos Servidores do Estado de Minas Gerais. ${ }^{3}$ Doutora em Medicamentos e Assistência Farmacêutica pela Universidade Federal de Minas Gerais. Professor do Departamento de Enfermagem Básica da Escola de Enfermagem da UFMG. ${ }^{4}$ Doutorado em Bioinformática pela Universidade Federal de Minas Gerais. Diretor de Assuntos Profissionais da Associação Mineira de Epidemiologia e Controle de Infecções. ${ }^{5}$ Doutor em Ciências pelo Instituto de Ciências Biológicas da UFMG. Professor do Departamento de Enfermagem Básica da Escola de Enfermagem da UFMG. ${ }^{6}$ Doutor em Enfermagem pela Escola de Enfermagem da UFMG. Professor do Departamento de Enfermagem da Escola de Enfermagem da UFMG.
} 


\section{INTRODUÇÃO}

As lesões por pressão (LPP) são eventos comuns, os quais persistem ao longo dos anos, acometendo pacientes hospitalizados e em cuidados domiciliares. Envolvem uma categoria de lesões, sendo na maioria das vezes evitáveis, cuja ocorrência implica no impacto na qualidade de vida do indivíduo e de sua família, como também nos altos custos para o sistema de saúde ${ }^{(1)}$.

A etiologia da lesão por pressão (LPP) é multifatorial incluindo os fatores intrínsecos e extrínsecos. São fatores intrínsecos aqueles pertencentes ao indivíduo tais como: idade, qualquer fator fisiológico como a presença de doenças crônicas e morbidades. Essas podem ser Hipertensão Arterial Sistêmica (HAS), Diabetes Mellitus (DM) ou doenças circulatórias, inconsciência, imobilidade, perda de sensibilidade ou da função motora, incontinência urinária ou fecal, desnutrição e índice de massa corporal muito alto ou muito baixo. Já os fatores extrínsecos são aqueles aos quais o indivíduo está exposto, como exemplo: a pressão de contato sobre a proeminência óssea, as forças de cisalhamento, a fricção e a umidade ${ }^{(2)}$.

As lesões por pressão podem desenvolverse em 24 horas ou levar até cinco dias para se manifestarem. Sendo a pressão o principal agente para a formação da LPP em indivíduos que ficam impossibilitados de se movimentar, os quais estão expostos a áreas de compressão excessivas envolvendo os tecidos macios contra as proeminências ósseas. Esse fato ocorre devido a uma pressão exercida na pele superior a pressão capilar normal $(32 \mathrm{mmHg})$, acarretando o colapso desses capilares, isto é, há uma diminuição da irrigação sanguínea, que leva o tecido à hipóxia e à redução dos nutrientes daquela região. Eventualmente, a área inflama pode chegar à morte celular e necrose celular local, desencadeando a formação da LPP. As localizações mais frequentes para $O$ desenvolvimento das lesões são em áreas onde a pressão do corpo nas proeminências ósseas é maior, como região sacral, trocantérica, isquiática e calcânea ${ }^{(3)}$.

Conforme estabelecido pela NPUAP (2016) as lesões por pressão são estadiadas para indicar a extensão do dano tissular acometido. Desse modo, as lesões apresentam as seguintes definições: Estágio 1 (pele íntegra com eritema não branqueável); Estágio 2 (perda de espessura da pele com exposição da derme); Estágio 3 (perda total da espessura da pele); Estágio 4 (perda de espessura da pele e perda tissular); Lesão por pressão não estadiável; Lesão por pressão relacionada a Dispositivos Médicos ; Lesão por Pressão em Membrana Mucosa.

$O$ ideal a se fazer quando existe o risco do desenvolvimento de lesão por pressão é a prevenção. Visando isso, o Ministério da Saúde (MS), em parceria com a Agência de Vigilância Sanitária (ANVISA), instituiu em abril de 2013, com a Portaria no 529, o Programa Nacional de Segurança do Paciente (PNSP). Seu objetivo é a contribuição para a qualificação do cuidado em saúde em todos os estabelecimentos de saúde do território nacional, motivando melhorias relativas à segurança do paciente, de forma a prevenir e reduzir a incidência de eventos adversos no atendimento e na internação, sendo a LPP um dos eventos considerados. Uma das principais ações do PNSP é a obrigatoriedade dos hospitais e serviços de saúde possuírem um Núcleo de Segurança do Paciente (NSP) implementado ${ }^{(3)}$.

$O$ desenvolvimento de condutas que objetivem a prevenção de LPP deve ser meta de qualquer serviço de saúde. Os profissionais de saúde necessitam estar atentos inclusive às questões de gastos, pois um paciente afetado pela complicação implica em aumento de gastos para o estabelecimento.

De acordo com a literatura ${ }^{(4)}$, existe uma relação proporcional entre os custos e os estágios das lesões por pressão, de modo que, quanto mais severa a lesão, maior o gasto com o tratamento.

Diferentes coberturas apresentam custos que variam conforme a área e gravidade das LPP. Estudo sobre este tema ${ }^{(5)}$ apresentou custos envolvidos no tratamento das LPP. Tal trabalho pode fornecer subsídios para os tomadores de decisão, assim como para a realização de investigações sobre custo-efetividade, fator crucial na gestão do cuidado. Porém, mais estudos são necessários.

Apesar da conscientização dos profissionais da enfermagem sobre as consequências físicas e emocionais para os pacientes, a maioria deles desconhece as consequências econômicas para a instituição quando um paciente desenvolve este evento adverso: LPP. Esse foi considerado o problema desta pesquisa. Além disso, para realização da sistematização da assistência de enfermagem (SAE), a caracterização 
socioeconômica e epidemiológica do paciente que desenvolve esse agravo torna-se uma aliada para sua prevenção. Desta forma, este trabalho objetiva avaliar o custo do tratamento de LPP, relacionado a materiais de curativos e mão de obra do profissional de enfermagem, bem como caracterizar a população acometida de LPP durante a internação hospitalar.

Esse conhecimento irá contribuir para o planejamento adequado da assistência possibilitando o direcionamento do uso racional e eficiente de recursos muitas vezes escassos, colaborando principalmente para evitarem-se eventos adversos ao paciente.

\section{MÉTODO}

Trata-se de estudo transversal, de caráter descritivo, com dados secundários de análise de custos diretos com materiais de curativos no tratamento de lesões por pressão (LPP). Foi realizado em um hospital de médio porte, de ensino, no estado de Minas Gerais. Os participantes foram todos os pacientes que desenvolveram lesão por pressão por ocasião da internação no hospital cenário desta pesquisa, durante o primeiro semestre de 2018. Utilizou-se como critério de inclusão o paciente possuir idade igual ou superior a 18 anos e ter sido submetido a tratamento de LPP. A amostra foi não probabilística, totalizando 60 participantes.

Os dados foram coletados via formulário, nos prontuários dos pacientes que desenvolveram LPP, com preenchimento retrospectivo, abrangendo variáveis sociodemográficas, clínicas e relacionadas ao custo. Foi elaborado um instrumento de coleta de dados para realização da pesquisa.

As variáveis sociodemográficas foram idade, sexo, cor, situação laboral e estado civil. As variáveis clínicas foram doença de base (sistema acometido), tempo de internação e desfecho (alta, transferência ou óbito). As variáveis relacionadas ao custo foram materiais utilizados, gastos com o material empregado e hora custo do profissional de enfermagem (enfermeiro ou técnico de enfermagem) por troca de curativo e estabelecida pelo Conselho Regional de Enfermagem de Minas Gerais - COREn MG ( $\mathrm{R} \$ 37,91$ por hora).

Os dados coletados foram transferidos para planilhas do Microsoft $^{\circledR}$ Excel e, no primeiro momento, avaliados por meio de técnicas de estatística descritiva, cujo objetivo foi resumir as informações. A caracterização dos pacientes foi realizada por meio do cálculo das frequências absolutas e relativas das variáveis categóricas e cálculo de média, mediana, valor mínimo, máximo, desvio padrão e coeficiente de variação para variáveis quantitativas. O coeficiente de variação (CV), a relação entre o desvio padrão e a média, avalia o grau de heterogeneidade de cada variável quantitativa, mede o nível de variabilidade dos dados: se $\mathrm{CV}>30 \%$ então a variável apresenta grande variabilidade. Variável com CV > 100\%, quando o desvio padrão é maior que a média, tem variabilidade extremamente elevada. Para cada item consumido/exigido para tratamento de lesão por pressão, foi calculado o valor de referência para o seu custo. $O$ valor de referência ou "padrão de normalidade" foi definido como o percentil $90\left(p_{90}\right)$ do custo com cada componente do tratamento da úlcera de pressão: somente $10 \%$ dos pacientes têm gastos acima do percentil 90. Além disto, foram construídos diagramas de dispersão cruzando variáveis quantitativas com os custos, total e com materiais.

Em seguida, após a análise descritiva dos dados, foram realizadas análises univariadas para identificação dos fatores ao custo total e ao custo com materiais usados no tratamento para tratamento de lesão por pressão. Foram feitos testes de hipóteses estatísticos bilaterais, considerando um nível de significância de $5 \%$ ( $\alpha=$ 0.05). A análise univariada foi conduzida para avaliar a associação bruta das variáveis independentes de interesse com os desfechos de custo. Variáveis categóricas foram avaliadas por meio do teste de qui-quadrado de Pearson e por testes exatos (quando necessário); variáveis quantitativas foram avaliadas por teste não paramétrico de Mann-Whitney ou Kruskal-Wallis.

$\mathrm{Na}$ última etapa foi feita análise multivariada por meio de regressão linear múltipla para o desfecho custo total. As variáveis que compuseram o modelo nesta etapa da análise multivariada foram selecionadas por meio da análise univariada (valor- $p \leq 0,25$ ). Para a seleção dos fatores independentes associadas ao custo total foram aplicados métodos de regressão linear múltipla com seleção automática de variáveis ou Foward Stepwise. Três metodologias de Foward Stepwise foram usadas: Foward Stepwise Wald, Conditional Foward Stepwise e Foward Stepwise. A aplicabilidade do modelo de regressão linear múltipla foi avaliada 
pelo coeficiente de determinação $\left(r^{2}\right)$. Todas as análises foram feitas pelo programa Statistical Package for the Social Sciences ${ }^{\circledR}$ (SPSS), versão 18.0 e pelo próprio Microsoft ${ }^{\circledR}$ Excel.

O projeto foi submetido ao Comitê de Ética em Pesquisa (COEP) da Universidade Federal de Minas Gerais (UFMG), tendo sido aprovado sob número 2930479. Foi solicitada dispensa do Termo de Consentimento Livre Esclarecido (TCLE), uma vez que os dados foram retrospectivos e coletados em prontuários e nos custos liberados pela Farmácia do hospital. O anonimato dos pacientes e da instituição foram preservados. Os riscos foram mínimos, uma vez que somente os pesquisadores tiveram acesso aos dados, tanto dos prontuários quanto dos custos liberados pela Farmácia.

\section{RESULTADOS E DISCUSSÃO}

Os resultados e a discussão estão apresentados em três etapas, denominadas Parte 1, Parte 2 e Parte 3. A Parte 1 refere-se ao "Perfil dos pacientes analisados". A Parte 2 diz respeito ao "Perfil de uso de materiais de curativo e custos". A Parte 3 intitula-se "Fatores associados aos custos para o tratamento de lesões por pressão". Os dados estão apresentados em gráficos, tabelas e diagramas.

\section{Parte 1 - Perfil de pacientes}

A maioria dos pacientes (63\%) era do sexo feminino. Esse dado também foi encontrado em estudo sobre LPP em pacientes $\operatorname{críticos}^{(6)}$, no qual $51 \%$ eram mulheres. No entanto, em pesquisa a respeito da incidência de $\operatorname{LPP}^{(7)}$, esse agravo acometeu mais os homens (52,9\%). Apesar de esses dois estudos terem sido direcionados para pacientes internados em terapia intensiva, essa diferença entre ambos pode estar relacionada às comorbidades de cada paciente.

Quanto à cor, $62 \%$ dos pacientes eram de cor branca, enquanto que os pardos e negros, juntos, constituíram $38 \%$ da casuística. A literatura não é unânime nesse aspecto, uma vez que estudo sobre incidência de LPP em pacientes internados teve como maioria (51\%) pacientes de cor parda ou negra. No entanto, estudiosos postulam que a pele negra é mais resistente à agressão externa causada pela umidade e fricção ${ }^{(9)}$.

Em relação à situação laboral, $47 \%$ estavam aposentados, enquanto $41 \%$ trabalhavam fora. Ressalta-se que a média de idade neste trabalho foi de 78 anos, ou seja, idade compatível com aposentadoria. No entanto, em pesquisa sobre $\operatorname{LPP}^{(6)}, 55 \%$ dos participantes ainda estavam ativos no trabalho. A não consideração dos afazeres domésticos como trabalho não foi característica deste estudo, uma vez que $13 \%$ dos pacientes se identificaram como trabalhadores do lar.

No que tange ao estado civil, $45 \%$ estavam casados. Os demais eram viúvos (37\%), solteiros (15\%) ou separados (3\%). Dado diferente foi encontrado em literatura ${ }^{(10)}$ sobre perfil dos pacientes com lesão por pressão, onde $52 \%$ eram solteiros e $\mathbf{2 7 \%}$ eram casados. Este dado pode sofrer variações de acordo com a idade da casuística. Os autores lembram que os cônjuges se tornam os cuidadores de seus maridos e esposas, demonstrando preocupação em proporcionar uma boa assistência de saúde para seus companheiros.

As doenças de base mais frequentes foram, por sistema acometido, sistema neurológico $(60 \%)$, sistema cardiovascular $(47 \%)$, sistema locomotor (47\%), sistema endócrino (43\%), sistema geniturinário (27\%), sistema respiratório (18\%) e sistema digestório (15\%). Os demais (outros) constituíram 18\%. A literatura varia nesse aspecto, uma vez que autores observaram, em estudo sobre LPP, que $63 \%$ dos agravos associados eram doenças infecciosas, parasitárias e neoplasias ${ }^{(11)}$. Lembra-se que infecções e neoplasias podem cursar com outros fatores de risco para LPP.

A idade média dos pacientes deste estudo foi 78 anos, com desvio padrão de 14,3. Em relação à pessoa idosa, considera-se que as LPP são complicações possíveis de ocorrer em pessoas em situação de fragilidade, principalmente naquelas com restrição de mobilidade e idade avançada ${ }^{(12)}$. A maioria absoluta dos pacientes avaliados (72\%) tinha idade entre 70 e 95 anos. A idade variou de 35 a 100 anos.

O peso dos pacientes por ocasião da internação foi em média $64 \mathrm{Kg}$, com desvio padrão de 12,5 KG. Não foram registrados nos prontuários dados referentes à altura dos pacientes, impossibilitando o cálculo de Índice de Massa Corporal (IMC). Indivíduos que pesavam menos de $30 \mathrm{Kg}$ ou peso entre 30 e $40 \mathrm{Kg}$ constituíram juntos $5 \%$ da casuística. Em contrapartida, o peso entre 80 e $90 \mathrm{Kg}$ acometeu $2 \%$ da amostra. Reportando-se a literatura ${ }^{(13)}$, há 
associação entre déficit nutricional e desenvolvimento de LPP e retardo da cicatrização. No entanto, revisão integrativa de literatura mostrou existir uma taxa elevada de LPP em pacientes críticos com peso adequado e/ ou com sobrepeso ${ }^{(14)}$. Neste trabalho, a maioria absoluta (30\%) dos pacientes apresentava peso entre 50 e $60 \mathrm{Kg}$,

Quanto ao tempo de tratamento, a taxa de internação em semanas variou de uma a mais de 20 semanas. A maioria dos sujeitos avaliados (62\%) teve tratamento realizado em até três semanas. Entretanto, observou-se uma grande heterogeneidade nos resultados. Alguns pacientes $(12 \%)$ ficaram internados por mais de 15 semanas. Essa variação de tempo pode estar relacionada a uma série de fatores, como doença de base e idade, dentre outros. Em revisão integrativa sobre cuidados de enfermagem na prevenção de LPP, especificamente para os internados em $\mathrm{CTI}^{(15)}$, os autores selecionaram 18 artigos que citavam fatores de risco para desenvolvimento desse agravo. No entanto, apenas um artigo indicou o tempo de internação como fator de risco para LPP. Ainda assim, sabese que a internação pode estar atrelada a uma série de fatores, como diminuição da mobilidade, drogas vasoativas, cateter vesical de demora, ventilação mecânica, fricção e cisalhamento, umidade, doenças cardiovasculares ou de sistema nervoso e outras ${ }^{(15)}$.

Os dados clínicos dos pacientes e o tempo de internação e tratamento encontram-se na Tabela 1.

Tabela 1. Perfil clínico dos pacientes avaliados - variáveis quantitativas.

\begin{tabular}{|c|c|c|c|c|}
\hline Variável & Média & Mediana & $\begin{array}{l}\text { Desvio } \\
\text { padrão }\end{array}$ & $\begin{array}{l}\text { Coeficiente de } \\
\text { variação }\end{array}$ \\
\hline Idade (anos) & 78 & 81 & 14,3 & $18 \%$ \\
\hline Peso ao se internar (Kg) & 64 & 65 & 12,5 & $20 \%$ \\
\hline Tempo total de tratamento (semanas) & 4 & 2 & 4,9 & $125 \%$ \\
\hline Tempo total de internação (semanas) & 6 & 3 & 8,9 & $142 \%$ \\
\hline
\end{tabular}

O coeficiente de variação, a relação entre o desvio padrão e a média mostram uma variação muito grande nos dados quando assumem valores acima de $30 \%$. Se a média apresenta valor muito diferente da mediana, então, há presença de dados aberrantes e a mediana deve ser escolhida para representar os dados.

Em relação ao desfecho da internação, chamou-nos a atenção a taxa de óbitos: $50 \%$. Dos demais, $38 \%$ tiveram alta e $12 \%$ foram transferidos para outras instituições. Relembra-se que $60 \%$ dos pacientes tinham agravos neurológicos, o que pode ter-lhes conferido um prognóstico mais reservado.

\section{Parte 2 - Perfil de uso de materiais de curativo e custos}

Nos dados sobre o custo com materiais de curativo e mão de obra, faz-se importante ressaltar que as coberturas e medicamentos tópicos utilizados nesse serviço são produtos indicados pela literatura atual ${ }^{(10)}$. São utilizados: placa de hidrocolóide, hidrogel, alginato de cálcio ou de sódio, tela impregnada com petrolato, carvão ativado, sulfadiazina de prata, colagenase, filme transparente, reservatório para curativo a vácuo e kit curativo pressão negativa, além de materiais básicos como ataduras, compressas de gaze e soro fisiológico. Observou-se grande heterogeneidade em todos os itens de uso no tratamento de lesões por pressão (Tabela 2):

Tabela 2 - Perfil de custo com materiais de curativo e mão de obra

\begin{tabular}{|c|c|c|c|c|c|c|c|}
\hline $\begin{array}{l}\text { Item de custo para tratamento de } \\
\text { lesão por pressão }\end{array}$ & Soma & Mínimo & Máximo & Média & Mediana & $\begin{array}{l}\text { Desvio } \\
\text { padrão }\end{array}$ & $\begin{array}{l}\text { Coeficiente de } \\
\text { variação }\end{array}$ \\
\hline Hidrocoloide $20 \times 20 \mathrm{~cm}$ valor total & $\mathrm{R} \$ 535$ & $\mathrm{R} \$ 0$ & $\mathrm{R} \$ 170$ & $\mathrm{R} \$ 9$ & $\mathrm{R} \$ 0$ & $R \$ 30$ & $333 \%$ \\
\hline Agulha desc $25 \times 8$ valor total & $\mathrm{R} \$ 195$ & $\mathrm{R} \$ 0$ & $\mathrm{R} \$ 27$ & $\mathrm{R} \$ 3$ & $\mathrm{R} \$ 1$ & $\mathrm{R} \$ 5$ & $152 \%$ \\
\hline Age valor total & $\mathrm{R} \$ 1.242$ & $\mathrm{R} \$ 0$ & $\mathrm{R} \$ 347$ & $\mathrm{R} \$ 21$ & $\mathrm{R} \$ 4$ & $\mathrm{R} \$ 60$ & $289 \%$ \\
\hline
\end{tabular}




\begin{tabular}{|c|c|c|c|c|c|c|c|}
\hline Hidrogel valor total & $\mathrm{R} \$ 8.315$ & $\mathrm{R} \$ 0$ & $\mathrm{R} \$ 1.237$ & $\mathrm{R} \$ 139$ & $\mathrm{R} \$ 25$ & $\mathrm{R} \$ 258$ & $186 \%$ \\
\hline $\begin{array}{l}\text { Alginato alginato de cálcio/ sódio } \\
10 \times 10 \mathrm{~cm} \text { valor total }\end{array}$ & $\mathrm{R} \$ 4.363$ & $\mathrm{R} \$ 0$ & $\mathrm{R} \$ 1.814$ & $\mathrm{R} \$ 73$ & $\mathrm{R} \$ 0$ & $\mathrm{R} \$ 280$ & $385 \%$ \\
\hline $\begin{array}{l}\text { Tela impregnada com petrolatum } \\
7,5 \times 20 \mathrm{~cm} \text { valor total }\end{array}$ & $\mathrm{R} \$ 18$ & $\mathrm{R} \$ 0$ & $\mathrm{R} \$ 14$ & $\mathrm{R} \$ 0$ & $\mathrm{R} \$ 0$ & $\mathrm{R} \$ 2$ & $609 \%$ \\
\hline Alginato fita/cordão valor total & $\mathrm{R} \$ 372$ & $R \$ 0$ & $\mathrm{R} \$ 169$ & $R \$ 6$ & $\mathrm{R} \$ 0$ & $\mathrm{R} \$ 27$ & $429 \%$ \\
\hline Atadura $15 \mathrm{~cm} \times 1,8 \mathrm{~m}$ valor total & $R \$ 2.276$ & $R \$ 0$ & $\mathrm{R} \$ 442$ & $R \$ 38$ & $R \$ 7$ & $\mathrm{R} \$ 79$ & $208 \%$ \\
\hline Atadura $10 \mathrm{~cm} \times 1,8 \mathrm{~m}$ valor total & $\mathrm{R} \$ 1.036$ & $\mathrm{R} \$ 0$ & $\mathrm{R} \$ 277$ & $\mathrm{R} \$ 17$ & $\mathrm{R} \$ 5$ & $\mathrm{R} \$ 41$ & $240 \%$ \\
\hline $\begin{array}{l}\text { COMPRESSA GAZE } 7,5 \times 7,5 \quad \text { CM } \\
\text { VALOR TOTAL }\end{array}$ & $\mathrm{R} \$ 13.997$ & $\mathrm{R} \$ 2$ & $\mathrm{R} \$ 4.143$ & $\mathrm{R} \$ 233$ & $\mathrm{R} \$ 54$ & $\mathrm{R} \$ 568$ & $244 \%$ \\
\hline Soro fisiológico $100 \mathrm{ml}$ valor total & $R \$ 5.263$ & $\mathrm{R} \$ 4$ & $\mathrm{R} \$ 436$ & $R \$ 88$ & $\mathrm{R} \$ 42$ & $\mathrm{R} \$ 112$ & $127 \%$ \\
\hline Soro fisiológico $250 \mathrm{ml}$ valor total & $\mathrm{R} \$ 1.121$ & $R \$ 0$ & $\mathrm{R} \$ 265$ & $\mathrm{R} \$ 19$ & $R \$ 0$ & $\mathrm{R} \$ 47$ & $250 \%$ \\
\hline Carvão ativado valor total & $\mathrm{R} \$ 634$ & $\mathrm{R} \$ 0$ & $\mathrm{R} \$ 516$ & $\mathrm{R} \$ 11$ & $\mathrm{R} \$ 0$ & $\mathrm{R} \$ 67$ & $634 \%$ \\
\hline Sulfadiazina de prata valor total & $\mathrm{R} \$ 732$ & $\mathrm{R} \$ 0$ & $\mathrm{R} \$ 704$ & $\mathrm{R} \$ 12$ & $R \$ 0$ & $\mathrm{R} \$ 91$ & $746 \%$ \\
\hline Colagenase valor total & $\mathrm{R} \$ 4.370$ & $\mathrm{R} \$ 0$ & $\mathrm{R} \$ 1.102$ & $R \$ 73$ & $R \$ 0$ & $\mathrm{R} \$ 190$ & $260 \%$ \\
\hline $\begin{array}{l}\text { Filme transparente } 10 \mathrm{~cm} \times 12 \mathrm{~cm} \\
\text { valor total }\end{array}$ & $\mathrm{R} \$ 1.340$ & $R \$ 0$ & $\mathrm{R} \$ 523$ & $\mathrm{R} \$ 22$ & $\mathrm{R} \$ 0$ & $\mathrm{R} \$ 71$ & $317 \%$ \\
\hline Kit curativo pressao neg. Valor total & $R \$ 3.245$ & $\mathrm{R} \$ 0$ & $\mathrm{R} \$ 2.445$ & $R \$ 54$ & $\mathrm{R} \$ 0$ & $\mathrm{R} \$ 330$ & $611 \%$ \\
\hline $\begin{array}{l}\text { Reservatorio para curativo a vacuo } \\
\text { valor total }\end{array}$ & $\mathrm{R} \$ 1.959$ & $R \$ 0$ & $\mathrm{R} \$ 1.123$ & $R \$ 33$ & $R \$ 0$ & $\mathrm{R} \$ 179$ & $549 \%$ \\
\hline Gasto total com materiais & $\mathrm{R} \$ 51.014$ & $R \$ 9$ & $\mathrm{R} \$ 8.388$ & $\mathrm{R} \$ 850$ & $R \$ 227$ & $\mathrm{R} \$ 1.667$ & $196 \%$ \\
\hline $\begin{array}{l}\text { Hora custo do } \\
\text { (enfermeiro/técnico } \\
\text { enfermagem) }\end{array}$ & $\mathrm{R} \$ 62.172$ & $\mathrm{R} \$ 76$ & $R \$ 6.141$ & $\mathrm{R} \$ 1.036$ & $\mathrm{R} \$ 455$ & $\mathrm{R} \$ 1.293$ & $125 \%$ \\
\hline Custo total & $\mathrm{R} \$ 113.186$ & $\mathrm{R} \$ 123$ & $\mathrm{R} \$ 13.998$ & $\mathrm{R} \$ 1.886$ & $\mathrm{R} \$ 700$ & $\mathrm{R} \$ 2.665$ & $141 \%$ \\
\hline
\end{tabular}

Observa-se que a Tabela 2 apresenta grande heterogeneidade em todos os itens de uso no tratamento de LPP, indicando a soma de cada produto utilizado durante o primeiro semestre de 2018. Tomando-se como exemplo o hidrogel, os gastos no semestre com esse produto foram de $\mathrm{R} \$ 8.315,00$.

Em contrapartida, os gastos com tela impregnada com petrolato foram de $\mathrm{R} \$ 18,00$ ao todo. Importante ressaltar que produtos essencialmente básicos podem ter um preço único razoável, entretanto, são utilizados em larga escala, como o soro fisiológico de $100 \mathrm{ml}$, cujo mínimo neste trabalho foi de $\mathrm{R} \$ 4,00$ e máximo de $\mathrm{R} \$ 436,00$, numa média de $\mathrm{R} \$ 88,00$ por paciente, com desvio padrão de $\mathrm{R} \$ 112,00$. Considera-se que o tratamento de qualquer lesão de pele, utilizando-se coberturas e materiais de ponta é justificado, levando-se em consideração os benefícios de uma cicatrização mais rápida e menos sofrida para o paciente e de um trabalho sistematizado dos profissionais.

Em estudo observacional ${ }^{(10)}$ realizado na unidade de cuidados paliativos de hospital de
Minas Gerais, cuja casuística foi de 40 pacientes, os autores observaram que os gastos com os materiais para o tratamento de LPP ultrapassava $\mathrm{R} \$ 1.220,00$ diários, perpassando a $\mathrm{R} \$ 36.629,95$ mensais, totalizando um gasto de $\mathrm{R} \$ 445.664,38$ anual. Esses valores são exclusivos dos materiais, utilizados para o tratamento de LPP, uma vez que os autores não mencionam gastos com mão de obra dos profissionais. Assim, infere-se que o tratamento de LPP é por deveras oneroso à instituição. Medidas de prevenção da LPP precisam ser intensificadas, visando inclusive a diminuição de gastos.

Este trabalho buscou o valor dos custos diretos com materiais de curativo (média e mediana, intervalo de confiança 95\%) e os custos indiretos, que se referem à realização do curativo por técnico de enfermagem ou por enfermeiro. $O$ COREn MG estabelece o valor de R\$37,91 para cada hora dedicada à realização de curativo, tanto para o técnico de enfermagem quanto para o enfermeiro. Neste trabalho, o valor médio deste atendimento foi de $\mathrm{R} \$ 1.036,00$. A maioria dos pacientes avaliados (68\%) teve custo máximo 
de $\mathrm{R} \$ 1.000,00$. Há grande disparidade nos resultados, com alguns poucos pacientes (10\%) com custo com mão de obra acima de R\$ $2.500,00$.

A literatura ${ }^{(5)}$ apresenta cálculos de mão de obra baseados na folha salarial de todos os funcionários envolvidos via Sistema de Apuração do Ponto do Trabalhador. Para os autores, houve uma variação de 5,75 a 33,73 minutos na aplicação de todas as coberturas. Os autores comparam os curativos das LPP sacrais com as demais, levando-se em conta que o tempo para posicionar a pessoa atendida, bem como para realizar a limpeza das LPP sacrais, foram maiores.

A seguir, apresentam-se custos máximos de referência, baseados no percentil 90 de custos com materiais de curativo e mão de obra para o tratamento de lesões por pressão (Tabela 3).

Tabela 3 - Custos máximos de referência para o tratamento de lesões por pressão.

\begin{tabular}{ll}
\hline Item de custo para tratamento de lesão por pressão & $\begin{array}{l}\text { Custo máximo de referência } \\
\text { (percentil 90) }\end{array}$ \\
\hline Hidrocoloide $20 \times 20 \mathrm{~cm}$ valor total & $\mathrm{R} \$ 4$ \\
Agulha desc $25 \times 8$ valor total & $\mathrm{R} \$ 7$ \\
Age valor total & $\mathrm{R} \$ 35$ \\
Hidrogel valor total & $\mathrm{R} \$ 433$ \\
Alginato alginato de cálcio/ sódio $10 \times 10 \mathrm{~cm}$ valor total & $\mathrm{R} \$ 54$ \\
Tela impregnada com petrolatum $7,5 \times 20 \mathrm{~cm}$ valor total & $\mathrm{R} \$ 0$ \\
Alginato fita/cordão valor total & $\mathrm{R} \$ 0$ \\
Atadura 15cmx1,8m valor total & $\mathrm{R} \$ 81$ \\
Atadura 10cm $\times 1,8 \mathrm{~m}$ valor total & $\mathrm{R} \$ 36$ \\
COMPRESSA GAZE 7,5x7,5 CM VALOR TOTAL & $\mathrm{R} \$ 517$ \\
Soro fisiológico 100 ml valor total & $\mathrm{R} \$ 292$ \\
Soro fisiológico 250 ml valor total & $\mathrm{R} \$ 78$ \\
Carvão ativado valor total & $\mathrm{R} \$ 0$ \\
Sulfadiazina de prata valor total & $\mathrm{R} \$ 0$ \\
Colagenase valor total & $\mathrm{R} \$ 158$ \\
Filme transparente $10 \mathrm{~cm} \times 12 \mathrm{~cm}$ valor total & $\mathrm{R} \$ 48$ \\
Kit curativo pressao neg. Valor total & $\mathrm{R} \$ 0$ \\
Reservatorio para curativo a vacuo valor total & $\mathrm{R} \$ 0$ \\
Gasto total com materiais & $\mathrm{R} \$ 2.128$ \\
Hora custo do profissional (enfermeiro/técnico de enfermagem) & $\mathrm{R} \$ 2.438$
\end{tabular}

Somente $10 \%$ dos pacientes apresentam custo acima do percentil 90, definido como o custo máximo de referência.

Em investigação a respeito dos custos de tratamento de LPP $^{(16)}$ foram mapeadas in loco as atividades de 26 curativos realizados por enfermeiras, em hospital público de Minas Gerais. Desses curativos, quatro correspondiam à LPP categoria 2; um à categoria 3; 13 à categoria 4; seis a LPP que não podem ser classificadas e outros dois não tiveram a categoria mencionada. Os autores estratificam os preços de coberturas e de adjuvantes, sendo eles: hidrocolóide; alginato; hidrofibra e prata; colágeno e alginato; hidrogel; prata nanocristalina. Considerando-se os custos com as coberturas, materiais médico-hospitalares e recursos humanos, os custos variaram de R\$ 16,41 a R\$ 260,18, dependendo do tipo de cobertura e extensão da LPP.

Dos pacientes avaliados neste trabalho, $38 \%$ tiveram custo máximo de $\mathrm{R} \$ 300$. Houve grande heterogeneidade nos resultados, com alguns poucos pacientes (11\%) com custo com materiais acima de $\mathrm{R} \$ 2.500$.

\section{Parte 3 - Fatores associados aos custos para o tratamento de lesões por pressão}

A comorbidade "Sistema Neurológico" apresentou custo significativo menor, quando presente $(p=0,001)$. Esses índices diferem dos que foram encontrados em trabalho sobre custos da LPP com pacientes neurocirúrgicos ${ }^{(16)}$. Ainda que não tenha sido realizado cálculo de associação, os autores observaram quatro 
pacientes, sendo que um dos pacientes com LPP estágio 2 necessitou fazer desbridamento com hidrogel com alginato de cálcio, o que aumentou o custo seu tratamento. No mesmo estudo, um dos pacientes, considerado o paciente mais crítico da casuística, foi admitido com LPP estágio 1 , mas evoluiu rapidamente para estágio 3 e necessitou fazer desbridamento cirúrgico, além do curativo de hidrogel, carvão ativado e uso de antimicrobiano. Todos os pacientes reportados $^{(16)}$ eram da clínica de neurocirurgia. No entanto, os pacientes que receberam algum tipo de prevenção obtiveram os menores custos em relação aos outros. 0 custo médio diário do participante denominado paciente 4 superou o custo médio diário do paciente 1 em 45\%. Nas comorbidades envolvendo os demais sistemas não houve associação.

Pacientes com alta hospitalar também tiveram custo significativamente menor, comparado a pacientes que evoluíram para óbito ou Transferência para outro hospital $(p=0,005)$.

Tabela 4 - Análise univariada dos possíveis fatores associados ao custo com materiais de curativo para o tratamento de lesões por pressão.

\begin{tabular}{|c|c|c|c|c|c|}
\hline Variável & Categorias & $\mathbf{n}$ & $\begin{array}{l}\text { Custo médio } \\
\text { com materiais }\end{array}$ & $\begin{array}{l}\text { Desvio } \\
\text { padrão }\end{array}$ & valor-P \\
\hline \multirow[t]{2}{*}{ Sexo } & Feminino & 38 & $\mathrm{R} \$ 788$ & $\mathrm{R} \$ 1.853$ & 0,104 \\
\hline & Masculino & 22 & $R \$ 958$ & $\mathrm{R} \$ 1.317$ & \\
\hline \multirow[t]{3}{*}{ Cor } & Branco & 37 & $\mathrm{R} \$ 575$ & $\mathrm{R} \$ 997$ & 0,869 \\
\hline & Negro & 9 & $\mathrm{R} \$ 879$ & $\mathrm{R} \$ 1.050$ & \\
\hline & Pardo & 14 & $\mathrm{R} \$ 1.560$ & $R \$ 2.901$ & \\
\hline \multirow[t]{4}{*}{ Estado civil } & Casado & 27 & $\mathrm{R} \$ 1.062$ & $\mathrm{R} \$ 1.810$ & 0,062 \\
\hline & Viúvo & 22 & $\mathrm{R} \$ 329$ & $\mathrm{R} \$ 618$ & \\
\hline & Solteiro & 9 & $\mathrm{R} \$ 1.539$ & $\mathrm{R} \$ 2.717$ & \\
\hline & Divorciado/separado & 2 & $\mathrm{R} \$ 634$ & $\mathrm{R} \$ 224$ & \\
\hline \multirow[t]{2}{*}{ Comorbidade: Sistema cardiovascular } & Não & 32 & $\mathrm{R} \$ 791$ & $R \$ 1.961$ & 0,524 \\
\hline & Sim & 28 & $\mathrm{R} \$ 918$ & $R \$ 1.283$ & \\
\hline \multirow[t]{2}{*}{ Comorbidade: Sistema digestório } & Não & 51 & $\mathrm{R} \$ 905$ & $\mathrm{R} \$ 1.751$ & 0,379 \\
\hline & Sim & 9 & $\mathrm{R} \$ 539$ & $\mathrm{R} \$ 1.095$ & \\
\hline \multirow[t]{2}{*}{ Comorbidade: Sistema endócrino } & Não & 34 & $\mathrm{R} \$ 908$ & $\mathrm{R} \$ 1.937$ & 0,858 \\
\hline & Sim & 26 & $\mathrm{R} \$ 774$ & $\mathrm{R} \$ 1.262$ & \\
\hline \multirow[t]{2}{*}{ Comorbidade: Sistema geniturinário } & Não & 44 & $\mathrm{R} \$ 809$ & $\mathrm{R} \$ 1.741$ & 0,960 \\
\hline & Sim & 16 & $\mathrm{R} \$ 964$ & $\mathrm{R} \$ 1.488$ & \\
\hline \multirow[t]{2}{*}{ Comorbidade: Sistema locomotor } & Não & 32 & $\mathrm{R} \$ 1.009$ & $\mathrm{R} \$ 1.738$ & 0,328 \\
\hline & Sim & 28 & $R \$ 668$ & $\mathrm{R} \$ 1.592$ & \\
\hline \multirow[t]{2}{*}{ Comorbidade: Sistema neurológico } & Não & 24 & $\mathrm{R} \$ 1.375$ & $\mathrm{R} \$ 1.900$ & 0,001 \\
\hline & Sim & 36 & $\mathrm{R} \$ 500$ & $\mathrm{R} \$ 1.412$ & \\
\hline \multirow[t]{2}{*}{ Comorbidade: Sistema respiratório } & Não & 49 & $\mathrm{R} \$ 748$ & $\mathrm{R} \$ 1.486$ & 0,510 \\
\hline & Sim & 11 & $\mathrm{R} \$ 1.306$ & $\mathrm{R} \$ 2.347$ & \\
\hline \multirow[t]{2}{*}{ Comorbidade: Outros } & Não & 49 & $\mathrm{R} \$ 756$ & $\mathrm{R} \$ 1.458$ & 0,364 \\
\hline & Sim & 11 & $\mathrm{R} \$ 1.270$ & $\mathrm{R} \$ 2.438$ & \\
\hline \multirow[t]{3}{*}{ Desfecho da internação } & Óbito & 30 & $R \$ 954$ & $\mathrm{R} \$ 1.808$ & 0,005 \\
\hline & Alta & 23 & $\mathrm{R} \$ 549$ & $\mathrm{R} \$ 1.639$ & \\
\hline & Transferência para outro hospital & 7 & $\mathrm{R} \$ 1.396$ & $\mathrm{R} \$ 952$ & \\
\hline
\end{tabular}

Obs.: valor-p calculado pelo teste não paramétrico de Mann-Whitney ou Kruskal-Wallis.

Discutindo-se custos para o tratamento de lesões por pressão por meio de análise univariada, dos possíveis fatores associados ao custo com materiais de curativo para o tratamento de lesões por pressão, obteve-se que as variáveis sexo, cor, situação laboral e estado civil não apresentaram $p<0,005$, ou seja, não houve significância.

$\mathrm{Na}$ análise univariada dos possíveis fatores associados ao custo total com o tratamento de lesões por pressão, observou-se que o estado civil "Viúvo" apresentou custo significativo menor 
$(p=0,042)$. Além disto, pacientes com a comorbidade "Sistema Neurológico" apresentaram custo significativo menor, quando presente $(p=0,011)$. Assim como na análise do custo com materiais, pacientes com alta hospitalar também tiveram custo total significativamente menor $(p=0,010)$. Esses dados encontram-se na Tabela 5:

Tabela 5 - Análise univariada dos possíveis fatores associados ao custo total com o tratamento de lesão por pressão LPP.

\begin{tabular}{|c|c|c|c|c|c|}
\hline Variável & Categorias & $\mathbf{n}$ & $\begin{array}{l}\text { Custo médio } \\
\text { com materiais }\end{array}$ & $\begin{array}{l}\text { Desvio } \\
\text { padrão }\end{array}$ & valor-P \\
\hline \multirow[t]{2}{*}{ Sexo } & Feminino & 38 & $\mathrm{R} \$ 1.691$ & $\mathrm{R} \$ 2.909$ & 0,092 \\
\hline & Masculino & 22 & $\mathrm{R} \$ 2.224$ & $\mathrm{R} \$ 2.204$ & \\
\hline \multirow[t]{3}{*}{ Cor } & Branco & 37 & $\mathrm{R} \$ 1.617$ & $\mathrm{R} \$ 1.921$ & 0,996 \\
\hline & Negro & 9 & $R \$ 1.548$ & $\mathrm{R} \$ 1.656$ & \\
\hline & Pardo & 14 & $\mathrm{R} \$ 2.816$ & $\mathrm{R} \$ 4.372$ & \\
\hline \multirow[t]{6}{*}{ Situação laboral } & Aposentado & 28 & $R \$ 1.937$ & $\mathrm{R} \$ 2.363$ & 0,528 \\
\hline & Do lar & 1 & $\mathrm{R} \$ 4.223$ & - & \\
\hline & Comerciante & 8 & $\mathrm{R} \$ 2.189$ & $\mathrm{R} \$ 4.791$ & \\
\hline & Serralheiro & 1 & $R \$ 1.699$ & - & \\
\hline & Vendedor & 1 & $\mathrm{R} \$ 1.199$ & - & \\
\hline & Outros & 21 & $\mathrm{R} \$ 1.634$ & $\mathrm{R} \$ 2.232$ & \\
\hline \multirow[t]{4}{*}{ Estado civil } & Casado & 27 & $\mathrm{R} \$ 2.511$ & $\mathrm{R} \$ 3.085$ & 0,042 \\
\hline & Viúvo & 22 & $\mathrm{R} \$ 898$ & $\mathrm{R} \$ 1.254$ & \\
\hline & Solteiro & 9 & $\mathrm{R} \$ 2.461$ & $R \$ 3.576$ & \\
\hline & Divorciado/separado & 2 & $\mathrm{R} \$ 1.752$ & $\mathrm{R} \$ 1.162$ & \\
\hline \multirow[t]{2}{*}{ Comorbidade: Sistema cardiovascular } & Não & 32 & $\mathrm{R} \$ 2.018$ & $\mathrm{R} \$ 3.203$ & 0,917 \\
\hline & Sim & 28 & $\mathrm{R} \$ 1.736$ & $\mathrm{R} \$ 1.923$ & \\
\hline \multirow[t]{2}{*}{ Comorbidade: Sistema digestório } & Não & 51 & $\mathrm{R} \$ 2.041$ & $\mathrm{R} \$ 2.820$ & 0,315 \\
\hline & Sim & 9 & $\mathrm{R} \$ 1.010$ & $\mathrm{R} \$ 1.298$ & \\
\hline \multirow[t]{2}{*}{ Comorbidade: Sistema endócrino } & Não & 34 & $\mathrm{R} \$ 1.998$ & $\mathrm{R} \$ 3.045$ & 0,687 \\
\hline & Sim & 26 & $\mathrm{R} \$ 1.741$ & $\mathrm{R} \$ 2.119$ & \\
\hline \multirow[t]{2}{*}{ Comorbidade: Sistema geniturinário } & Não & 44 & $\mathrm{R} \$ 1.833$ & $\mathrm{R} \$ 2.810$ & 0,726 \\
\hline & Sim & 16 & $\mathrm{R} \$ 2.035$ & $\mathrm{R} \$ 2.296$ & \\
\hline \multirow[t]{2}{*}{ Comorbidade: Sistema locomotor } & Não & 32 & $\mathrm{R} \$ 2.045$ & $\mathrm{R} \$ 2.848$ & 0,635 \\
\hline & Sim & 28 & $\mathrm{R} \$ 1.705$ & $\mathrm{R} \$ 2.479$ & \\
\hline \multirow[t]{2}{*}{ Comorbidade: Sistema neurológico } & Não & 24 & $\mathrm{R} \$ 2.710$ & $\mathrm{R} \$ 3.104$ & 0,011 \\
\hline & Sim & 36 & $\mathrm{R} \$ 1.338$ & $\mathrm{R} \$ 2.206$ & \\
\hline \multirow[t]{2}{*}{ Comorbidade: Sistema respiratório } & Não & 49 & $\mathrm{R} \$ 1.551$ & $\mathrm{R} \$ 2.116$ & 0,316 \\
\hline & Sim & 11 & $\mathrm{R} \$ 3.381$ & $\mathrm{R} \$ 4.172$ & \\
\hline \multirow[t]{2}{*}{ Comorbidade: Outros } & Não & 49 & $\mathrm{R} \$ 1.741$ & $R \$ 2.520$ & 0,316 \\
\hline & Sim & 11 & $R \$ 2.535$ & $\mathrm{R} \$ 3.296$ & \\
\hline \multirow[t]{3}{*}{ Desfecho da internação } & Óbito & 30 & $\mathrm{R} \$ 1.806$ & $\mathrm{R} \$ 2.532$ & 0,010 \\
\hline & Alta & 23 & $\mathrm{R} \$ 1.620$ & $\mathrm{R} \$ 3.128$ & \\
\hline & Transferência para outro hospital & 7 & $\mathrm{R} \$ 3.107$ & $\mathrm{R} \$ 970$ & \\
\hline
\end{tabular}

Obs.: valor-p calculado pelo teste não paramétrico de Mann-Whitney ou Kruskal-Wallis.

Os diagramas de dispersão, avaliando a relação entre tempo de tratamento e período de internação versus custo com materiais e custo total sugerem forte correlação entre ambos, os tempos e os custos para o tratamento de lesões por pressão (Figura 1):

Figura 1 - Diagramas de dispersão tempo de tratamento e custo com materiais. 

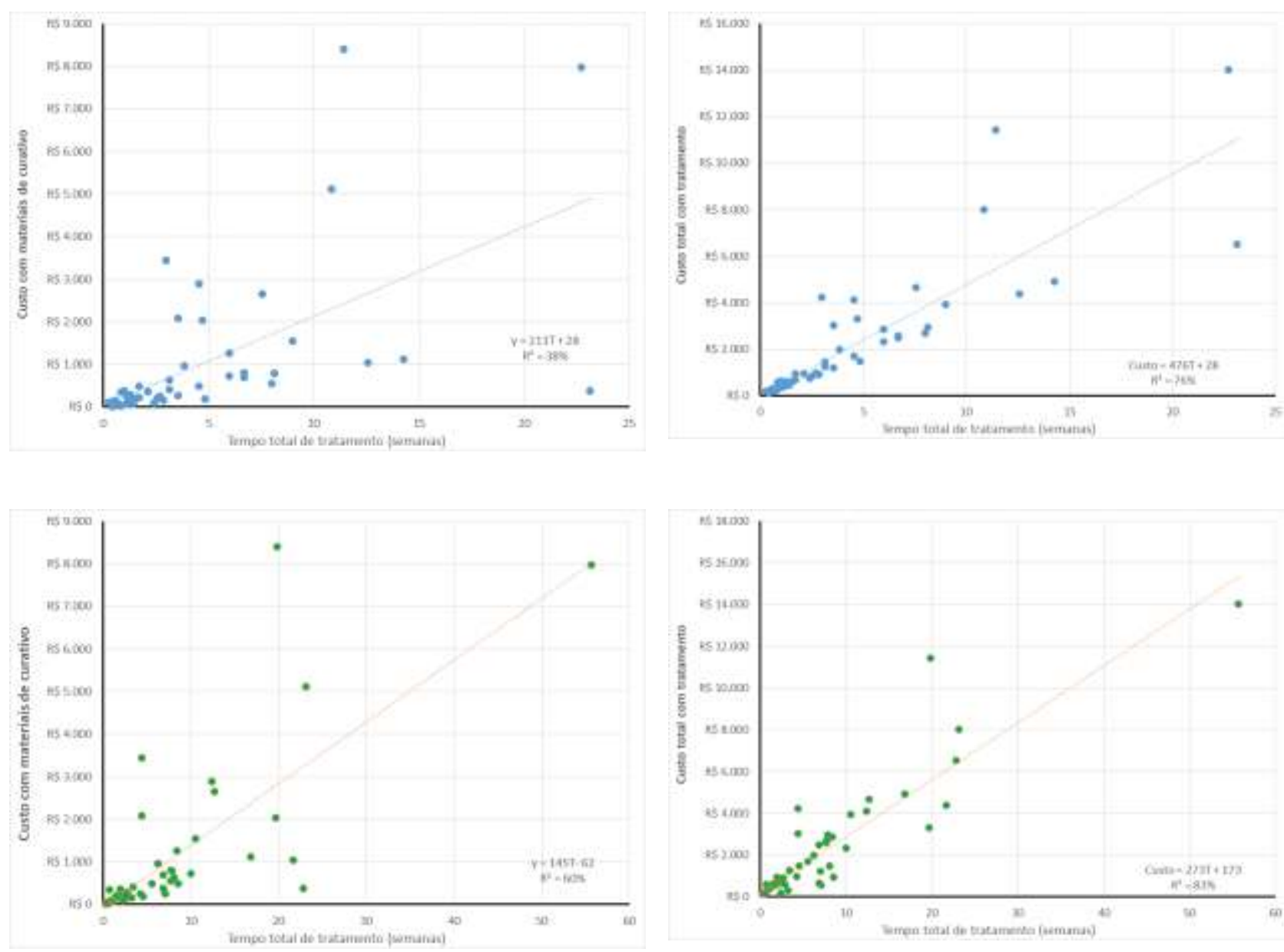

Fonte: Dados desta pesquisa

Há uma lógica nessa relação: maior tempo de internação implica em maior exposição aos fatores de risco. Segundo estudiosos ${ }^{(17)}$, a restrição ao leito, a dificuldade de mobilidade e a condição clínica favorecem o surgimento de LPP. Desse modo, há que se esforçar para minimizar os efeitos da internação hospitalar no que se refere a riscos de desenvolvimento de LPP.

No que diz respeito à análise multivariada dos possíveis fatores associados ao custo total para o tratamento de lesões por pressão considerando-se um conjunto de variáveis, observou-se que somente o tempo de internação e o tempo de tratamento mostraram-se significativamente associados ao custo total, com valor de p respectivamente 0,000 e 0,032 .

Tabela 6 - Análise multivariada para identificação de fatores associados ao custo total para tratamento da lesão por pressão.

\begin{tabular}{cccc}
\hline Variável & Coeficiente & Erro padrão & valor-P \\
\hline Tempo total de tratamento (semanas) & 166,1 & 58,4 & 0,006 \\
Tempo total de internação (semanas) & 192,8 & 32,0 & 0,000 \\
Constante & 25,8 & & \\
\hline
\end{tabular}

A avaliação por regressão linear múltipla foi obtida pelo método Stepwise Forward. As três metodologias de Foward Stepwise usadas (Foward Stepwise Wald, Conditional Foward Stepwise e Foward Stepwise) chegaram ao mesmo modelo. De todas as variáveis avaliadas, somente duas se associaram de forma independente e significativa com o custo total: tempo total de tratamento (semanas) e tempo total de internação (semanas).

O modelo multivariado pode ser usado para prever o custo total para o tratamento de LPP. Esse modelo utiliza o tempo de tratamento e o tempo de internação, sendo que eles têm um 
coeficiente de determinação $\left(r^{2}\right)$ de $85 \%$, ou seja, o modelo explica $85 \%$ da variabilidade no custo total de cada paciente.

Este trabalho teve como fator limitante a ausência nos prontuários de maiores informações sobre os pacientes, como por exemplo, a altura, o que possibilitaria o cálculo de IMC. Porém, os objetivos foram alcançados, permitindo se estimar o custo do tratamento de uma lesão por pressão em relação à sua prevenção.

\section{CONSIDERAÇÕES FINAIS}

Os resultados apresentados por este estudo mostraram, dentre outros aspectos, que a população de maior risco ao desenvolvimento das lesões por pressão foi a idosa, com média de idade de 78 anos, devido às alterações geradas pelo processo do envelhecimento, tornando a pele mais sensível, perda da musculatura e redução da gordura do tecido subcutâneo. Observou-se também que a maior parte dos pacientes é composta pelo sexo feminino (63\%), apresentando cor da pele com predominância na cor branca, $45 \%$ são casados, $47 \%$ aposentados, e $60 \%$ apresentam doenças de base relacionada ao sistema neurológico. O estado nutricional do paciente também contribui no processo da lesão por pressão, haja vista que pacientes desnutridos ou obesos são mais vulneráveis ao seu acometimento. No entanto, neste estudo a maioria (30\%) dos sujeitos analisados apresentava peso entre 50 e $60 \mathrm{Kg}$.

Em relação aos custos com tratamento de LPP, verificou-se a média de custo semestral do tratamento de LPP, por paciente, de $\mathrm{R} \$ 1.886,00$ e custo total semestral de $\mathrm{R} \$ 1_{113.186,00}$. Quanto à distribuição do custo com mão de obra envolvendo os profissionais da enfermagem (enfermeiros e técnicos de enfermagem) para o tratamento de LPP, a maioria dos pacientes avaliados (68\%) teve custo máximo de R\$ $1.000,00$, com alguns poucos pacientes (10\%) com custo com mão de obra acima de R\$ $2.500,00$. Os custos diretos foram apropriados com foco nos materiais e coberturas/adjuvantes utilizados e tempo de enfermagem investidos na realização desses procedimentos.

Este estudo contribuirá com a melhoria da gestão do atendimento em saúde, uma vez que proporcionará reflexões sobre a questão custo e benefícios da prevenção da LPP no tangente a redução de gastos, otimizando assim a prática de enfermagem. Ressalta-se ainda que o bem-estar e o conforto do paciente são fatores soberanos na gestão da qualidade da assistência de saúde.

\section{REFERÊNCIAS}

1. Campos MGCA, Souza ATO, Vasconcelos J MB, Lucena SAAP, Gomes SKA. Feridas complexas e estomias: aspectos preventivos e manejo clínico. João Pessoa: Ideia; 2016

2. Wada A, Teixeira NN, Ferreira M. Úlceras por pressão. Rev Med. 2010 [citado em 4 jan 2019]; 89(3-4):170-7. Disponível em: https://doi.org/10.11606/issn.16799836.v89i3/4p170-7

3. Domansky RC, Borges EL. Manual para prevenção de lesões de pele: recomendações baseadas em evidências. Rio de Janeiro: Rúbio; 2014.

4. Silva DRA, Bezerra SMG, Costa J P, Luz MHBA, Lopes VCA, Nogueira LT. Curativos de lesões por pressão em pacientes críticos: análise de custos. Rev Esc Enferm USP. 2017 [citado em 20 ago 2019]; 51(e03231):1-8. DOI: http://dx.doi.org/10.1590/S1980-

220X2016014803231

5. Andrade CCD, Almeida CFSC, Pereira WE, Alemão MM, Brandão C MR, Borges EL. Costs of topical treatment of pressure ulcer patients. Rev Esc Enferm USP. 2016 [citado em 3 abr. 2019]; 50(2):292-8. Disponível em: doi.org/10.1590/S0080-623420160000200016

6. Borghardt AT, Prado TN, Bicudo SDS, Castro DS, Bringuente MEO. Pressure ulcers in critically ill patients: incidence and associated factors. Ver. Bras. Enferm. 2016 [citado em 23 fev. 2019]; 69(3): 431-8. Disponível em: http://dx.doi.org/10.1590/0034-7167.2016690307i

7. Mendonça PK, Loureiro MDR, Ferreira Junior MA, Souza AS. Ocorrência e fatores de risco para lesões por pressão em centros de terapia intensiva. Ver. enferm. UFPE online. 2018 [citado em 13 dez 2018]; 12(2):303-11.

Disponível em https://doi.org/10.5205/19818963-v12i2a23251p303-311-2018

8. Matozinhos FP, Velasquez-Melendez G, Tiensoli SD, Moreira AD, Gomes FSL. Factors associated with the incidence of pressure ulcer during hospital stay. Rev Esc Enferm USP. 2017 [citado em 05 ago 2019]; 51: e03223. Disponível em https://dx.doi.org/10.1590/S1980$\underline{220 \times 2016015803223}$

9. Laranjeira CA, Loureiro S. Fatores de risco das úlceras de pressão em doentes internados num hospital português. Rev. salud 
pública. 2017 [citado em 05 ago 2019]; 19(1): 99-104. Disponível em: https://doi.org/10.15446/rsap.v19n1.42251 10. Costa AM, Matozinhos ACS, Trigueiro OS, Cunha RCG, Moreira L R. Custos do tratamento de úlceras por pressão em unidade de cuidados prolongados em uma instituição hospitalar de Minas Gerais. Rev. Enfermagem Revista. 2015 [citado em 7 abr. 2019]; 18(1): 58-74. Disponível em: http://periodicos.pucminas.br/index.php/enferm agemrevista/article/view/9378

11. Pacha HHP, Faria JIL, Oliveira KA, Beccaria LM. Lesão por Pressão em Unidade de Terapia Intensiva: estudo de caso-controle. Rev. Bras. Enferm. 2018 [citado em 5 ago 2019]; 71(6):3027-34. Disponível em: http://dx.doi.org/10.1590/0034-7167-2017-0950 12. Vieira VAS, Santos MDC, Almeida NA, Souza CC, Bernardes MFVG, Mata LRF. Risco de lesão por pressão em idosos com comprometimento na realização de atividades diárias. Rev. enferm. Cent.-Oeste Min. 2018 [citado em 5 ago 2019]; 8:e2599. Disponível em: http://dx.doi.org/10.19175/recom.v7i0.2599

13. Souza NR, Freire DA, Souza MAO, Melo JTS, Santos LV, Bushatsky M. Fatores predisponentes para o desenvolvimento da lesão por pressão em pacientes idosos: uma revisão integrativa. Estima. 2017 [citado em 5 ago 2019]; 15(4):229-39. Disponível em: http://dx.doi.org/ 10.5327/Z1806$\underline{3144201700040007}$

14. Sousa RG, Oliveira TL, Lima LR, Stival MM. Fatores associados à úlcera por pressão (UPP) em pacientes críticos: revisão integrativa da literatura Universitas: Ciências da Saúde. 2016 [citado em 5 ago. 2019]; 14(1)77-4. Disponível em http://dx.doi.org/ 10.5102/ucs.v14i1.3602

15. França JRG, Sousa BVN, Jesus V S. Cuidados de enfermagem na prevenção de lesões por pressão em Unidades de Terapia Intensiva: uma revisão sistemática. Revista Brasileira de Saúde Funcional. 2016 [citado em 16 jan. 2019];1(11): 16-31. Disponível em: http://www.seeradventista.com.br/ojs/index.php/RBSF/article/vie wFile/709/619

16. Lima ACB, Guerra DM. Avaliação do custo do tratamento de úlceras por pressão em pacientes hospitalizados usando curativos industrializados. Ciênc. saúde coletiva [online]. 2011 [citado em 9 mar 2019];16(1):267-77 Disponível em: http://www.scielo.br/scielo.php?script=sci artte xt\&pid=S1413-81232011000100029
17. Abreu MA, Rolim VE, Dantas RCO. Ações de enfermagem para prevenção de úlceras por pressão em clientes em unidade de terapia intensiva. RPI. 2017 [citado em 6 mar 2019]; (2):686-96. Disponível em: http://dx.doi.org/10.24219/rpi.v2i2.400

Nota: Este artigo é parte da monografia de especialização em Estomaterapia da Escola de Enfermagem da UFMG. Não há agência de fomento.

Recebido em: 07/06/2019

Aprovado em: 01/12/2019

Endereço de correspondência:

Miguir Terezinha V. Donoso

Endereço: Av. Alfredo Balena, 190, sala 206, Santa Efigênia. CEP: 30330.100 - Belo Horizonte/MG - Brasil E- mail: miguirdonoso@uol.com.br 